\title{
Networked Control Synthesis Using Time Delay Approach: State Feedback Case
}

\author{
Wrastawa Ridwan ${ }^{1}$, Bambang Riyanto Trilaksono ${ }^{2}$ \\ ${ }^{1}$ Department of Electrical Engineering, Gorontalo State University, Gorontalo, Indonesia \\ Jl. Jendral Sudirman no.6 Kota Gorontalo, Indonesia 96128 \\ ${ }^{2}$ School of Electrical Engineering \& Informatics, Bandung Institute of Technology, \\ Bandung, Indonesia, Jl. Ganeca no. 10 Bandung, Indonesia 40132 \\ r1space@yahoo.com, briyanto@lskk.ee.itb.ac.id
}

\begin{abstract}
Networked Control Systems (NCS) is a control system in which the sensors, controllers, and the actuators are connected via communication network. The use of communication network will lead to intermittent losses or delays of the information and may decrease the performance and cause instability. This paper presents the design of $H_{\infty}$ state feedback controller for NCS. The NCS is modelled as a time delay system. Two network features are considered: signal transmission delay and data packet dropout. Our objective is focused on the design of state feedback controller which guarantee asymptotic stability of the closed-loop systems. The proposed methods are given in the terms of Linear Matrix Inequality (LMI). Finally, we consider an unstable system for numerical example. It is shown that the state feedback controller proposed here make the closed-loop system stable with or without input disturbance.
\end{abstract}

Keywords: Networked Control Systems (NCS), Signal Transmission Delay, Data Packet Dropout, State Feedback Controller

\section{Introduction}

In modern control systems, physical plant, controller, sensors and actuator are difficult to be located at the same place, and hence these components need to be connected over network media. When feedback control system is closed via a communication channel, then the control system is classified as a Networked Control System (NCS) [1,2]. NCS has been attracted much attention due to significant advantages, such as reduced installation and maintenance cost, increased system agility, and so on [3]. There have many researcher to conduct research on the topic of NCS. To mention a few, [4] addressed the problem of quantized feedback control. Result on state feedback control could be seen in [5,6]. Stability analysis, and stabilization of NCS are investigated in [2,3] and the references therein. There are some parameters that arise when using communication network in NCS such as packet dropouts and signal transmission delay. The presence of these parameters can degrade the performance of the system and could lead to instability.

When information or energy is physically transmitted from one place to another, there is a delay associated with the transmission [7]. It is well known that the presence of time-delay is a source of instability [8]. Xia et al. [9] presents some basic theories of stability and synthesis of systems with time-delay, in the form of $\dot{x}(t)=A x(t)+A_{d} x(t-\tau(t))$, where $\tau(t)$ represents time-varying delay. Wu et al. [10] presents a method referred to as the free-weighting-matrix (FWM) approach for the stability analysis and control synthesis of various classes of timedelay systems. In [11], a new model for time delay systems is proposed, that is $\dot{x}(t)=A x(t)+A_{d} x\left(t-\tau_{1}(t)-\tau_{2}(t)\right)$. The new model is motivated by practical situation in Networked Control Systems (NCSs), where $\tau_{I}(t)$ is the time-delay from sensor to the controller and $\tau_{2}(t)$ is the time-delay from controller to the actuator. Based on such a system

Received: April 10 ${ }^{\text {th }}, 2011$. Accepted: December $20^{\text {th }}, 2011$ 
representation, [11] derived the stability condition. Gao et al. [5] presented a new stability condition and investigated the problem of $H_{\infty}$ performance analysis. Dey et al. [12] constructed a new Lyapunov-Krasovskii functional in obtaining the stability condition for such system, and provided less conservative delay upper bound, as compared to the conditions in [5,11].

Motivated by [12], in this paper we continue the preliminary result derived in [12] to the NCS problem. Our objective is focused on the design of $H_{\infty}$ state feedback controller for NCS. It is well known in systems and control community that $H_{\infty}$-norm constraint can be used to provide a prespecified disturbance attenuation level, and alternatively to analyze robust stability of dynamical system.

This paper improve the previous paper appeared in [16], that is reduce the number of variables in Theorem 1. In addition, analysis and discussion have been improved by exploring the effect of the parameters change.

Notation. The notation $X>0$ denotes a symmetric positive definite, asterisk (*) represents the elements of symmetric term in the symmetric block matrix. The superscripts " $T$ " and "- 1 " represent the transpose and inverse matrix, respectively. $L_{2}[0, \infty)$ is the space of square integrable functions on $[0, \infty)$.

Before proceeding further, we give the following lemmas.

Lemma 1. Schur Complement. Schur's formula says that the following statements are equivalent $[13,14]$ :

$$
\begin{array}{ll}
\text { i. } & \Phi=\left[\begin{array}{ll}
\Phi_{11} & \Phi_{12} \\
\Phi_{12}^{T} & \Phi_{22}
\end{array}\right]<0 \\
\text { ii. } & \Phi_{22}<0 \\
& \Phi_{11}-\Phi_{12} \Phi_{22}^{-1} \Phi_{12}^{T}<0
\end{array}
$$

Lemma 2. Consider the following system with two additive time-varying delays in the state [15]:

$$
\begin{aligned}
& \dot{x}(t)=A x(t)+A_{d} x\left(t-\tau_{1}(t)-\tau_{2}(t)\right)+E w(t), \\
& y(t)=C x(t)+C_{d} x\left(t-\tau_{1}(t)-\tau_{2}(t)\right)+F w(t), \\
& x(t)=\phi(t), \quad t \in[-\bar{\tau}, 0]
\end{aligned}
$$

Assumming that

$$
\tau_{1}(t) \equiv \bar{\tau}_{1}<\infty, 0 \leq \tau_{2}(t) \leq \bar{\tau}_{2}<\infty
$$

System (1) satisfying (2) either

(i). Asymptotically stable with $w=0$, or

(ii). Stable with $H_{\infty}$ disturbance attenuation level $\gamma(w \neq 0)$

if there exist matrices $P=P^{T}>0, \quad Q_{2}=Q_{2}^{T}>0, \quad R_{1}=R_{1}^{T}>0, \quad R_{2}=R_{2}{ }^{T}>0$, $R_{3}=R_{3}^{T}>0$ and $G_{\mathrm{i}}, L_{i}, M_{i}, N_{i}, i=1, \ldots, 4$ are free matrices satisfying,

$$
\left[\begin{array}{cc}
\Psi_{1}+\Psi_{2}+\Psi_{2}^{T}+\Psi_{3}+\Psi_{4}+\Psi_{6}^{T} \Psi_{6} & \Psi_{5} \\
* & \Xi_{5}
\end{array}\right]<0
$$


where,

$$
\begin{aligned}
& \Psi_{1}=\left[\begin{array}{ccccc}
Q_{2} & 0 & 0 & P & 0 \\
* & -Q_{2} & 0 & 0 & 0 \\
* & * & 0 & 0 & 0 \\
* & * & * & \bar{\tau} R_{1}+\bar{\tau}_{1} R_{2}+\bar{\tau}_{2} R_{3} & 0 \\
* & * & * & * & 0
\end{array}\right], \Psi_{5}=\left[\begin{array}{ccc}
L_{1} & M_{1} & N_{1} \\
L_{2} & M_{2} & N_{2} \\
L_{3} & M_{3} & N_{3} \\
L_{4} & M_{4} & N_{4} \\
0 & 0 & 0
\end{array}\right], \\
& \Psi_{2}=\Psi_{21} \Psi_{22}, \Psi_{21}=\left[\begin{array}{llllll}
A & 0 & A_{d} & -I & E
\end{array}\right]^{T}, \Psi_{22}=\left[\begin{array}{lllll}
G_{1}^{T} & G_{2}^{T} & G_{3}^{T} & G_{4}^{T} & 0
\end{array}\right] \\
& \Psi_{3}=\left[\begin{array}{ccccc}
L_{1}+L_{1}^{T}+M_{1}+M_{1}^{T} & L_{2}^{T}-M_{1}+M_{2}^{T}+N_{1} & -L_{1}+L_{3}^{T}+M_{3}^{T}-N_{1} & L_{4}^{T}+M_{4}^{T} & 0 \\
* & -M_{2}-M_{2}^{T}+N_{2}+N_{2}^{T} & -L_{2}-M_{3}^{T}-N_{2}+N_{3}^{T} & -M_{4}^{T}+N_{4}^{T} & 0 \\
* & * & -L_{3}-L_{3}^{T}-N_{3}-N_{3}^{T} & -L_{4}^{T}-N_{4}^{T} & 0 \\
* & * & * & 0 & 0 \\
* & * & * & * & 0
\end{array}\right], \\
& \left.\Psi_{4}=\operatorname{diag}\left\{0,0,0,0,-\gamma^{2} I\right\}, \Psi_{6}=\left[\begin{array}{lllll}
C & 0 & C_{d} & 0 & F
\end{array}\right], \Xi_{5}=\operatorname{diag} \mid-\bar{\tau}^{-1} R_{1},-\bar{\tau}_{1}^{-1} R_{2},-\bar{\tau}_{2}^{-1} R_{3}\right\}
\end{aligned}
$$

\section{System Description}

In this section, we model the NCS with consideration of the effect of network-induced delay and data packet dropout. Then we design the state feedback controller. The model of NCS follow the model described in [2]. We consider an NCS shown in Figure 1. Suppose the physical plant is given by the following system:

$$
\begin{aligned}
& \dot{x}(t)=A x(t)+B u(t)+E w(t) \\
& y(t)=C x(t)+D u(t)+F w(t)
\end{aligned}
$$

where $x(t) \in \mathfrak{R}^{n}$ is the state vector, $u(t) \in \mathfrak{R}^{p}$ is the control input, $y(t) \in \mathfrak{R}^{q}$ is the output vector and $w(t) \in \mathfrak{R}^{l}$ is the disturbance input which belongs to $L_{2}[0, \infty) ; A, B, E, C, D$, and $F$ are known system matrices with appropriate dimension.

It is assumed that sensor and sampler are clock-driven, while the controller, $\mathrm{ZOH}$ and actuator are event-driven. The sampling period is assumed to be $h$ (positive real constant) and the sampling instant are denoted as $s_{k}, k=1,2, \ldots$. The measurement of state variable is $\bar{x}$ and then transmitted with a single packet.

At the sampling instant $s_{k}$, we have,

$$
\bar{x}\left(s_{k}\right)=\left[\begin{array}{llll}
x_{1}\left(s_{k}\right) & x_{2}\left(s_{k}\right) & \ldots & x_{n}\left(s_{k}\right)
\end{array}\right]^{T}
$$

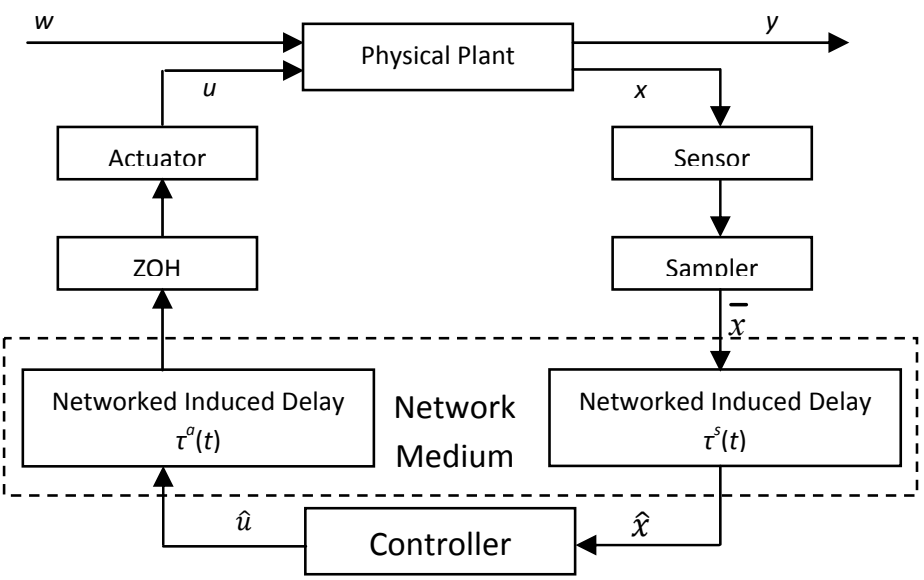

Figure 1. Networked control system 
Transmitted signal from the sampler to the controller experienced signal transmission delays $\tau_{k}^{s}$ that is

$$
\hat{x}\left(s_{k}\right)=\bar{x}\left(s_{k}-\tau_{k}^{s}\right)
$$

where $\tau_{k}^{S}$ is the network delay from the sampler to the controller at instant $k$.

Control signal from the controller is

$$
\hat{u}\left(s_{k}\right)=K \hat{x}\left(s_{k}\right)=K x\left(s_{k}-\tau_{k}^{s}\right)
$$

Then, transmitted signal from the controller to the $\mathrm{ZOH}$ experienced signal transmission delays that is

$$
u\left(s_{k}\right)=\hat{u}\left(s_{k}-\tau_{k}^{a}\right)
$$

where $\tau_{k}^{a}$ is the network delay from the controller to the $\mathrm{ZOH}$ at instant $k$.

Overall, transmitted signal from the sampler to $\mathrm{ZOH}$ experienced signal transmission delays $\eta_{k}$, that is

$$
\eta_{k}=\tau_{k}^{S}+\tau_{k}^{a}
$$

So that, the control signal is

$$
u\left(s_{k}\right)=K x\left(s_{k}-\tau_{k}^{S}-\tau_{k}^{a}\right)=K x\left(s_{k}-\eta_{k}\right)
$$

It is assumed that there are no delay between the sensor and the sampler and between the $\mathrm{ZOH}$ and the actuator.

We denote the updating instants of the $\mathrm{ZOH}$ as $t_{k}, k=1,2, \ldots$. The state feedback controller takes the following form:

$$
u\left(t_{k}\right)=K x\left(t_{k}-\eta_{k}\right)
$$

where $K$ is the state feedback gain.

Considering the behaviour of the $\mathrm{ZOH}$, the control input is

$$
u(t)=K x\left(t_{k}-\eta_{k}\right), \quad t_{k} \leq t<t_{k+1}
$$

From (4) and (8), we obtain the following closed-loop system:

$$
\begin{aligned}
& \dot{x}(t)=A x(t)+B K x\left(t_{k}-\eta_{k}\right)+E w(t), \\
& y(t)=C x(t)+D K x\left(t_{k}-\eta_{k}\right)+F w(t), \\
& t_{k} \leq t \leq t_{k+1}
\end{aligned}
$$

Network-induced delay always exists when the data transmits through a network and is nondifferentiable interval time-varying delay. So a natural assumption on $\eta_{k}$ can be made as $[4,5]$

$$
0<\eta_{m} \leq \eta_{k} \leq \eta_{M}
$$

where $\eta_{m}$ and $\eta_{M}$ denote the minimum and maximum delay bounds, respectively.

The effect of data packet dropouts in the communication channel can be described as the $\mathrm{ZOH}$ is not updated during the time interval of this event, which is referred to vacant sampling. Hence, the effect of one packet dropout in the transmission is just a case that one sampling period delay is induced in the updating interval of the $\mathrm{ZOH}[4,5]$. 


$$
t_{k+1}-t_{k}=\left(\delta_{k+1}+1\right) h+\eta_{k+1}-\eta_{k}
$$

where $\delta_{k+1}$ is the number of accumulated packet dropouts in the period.

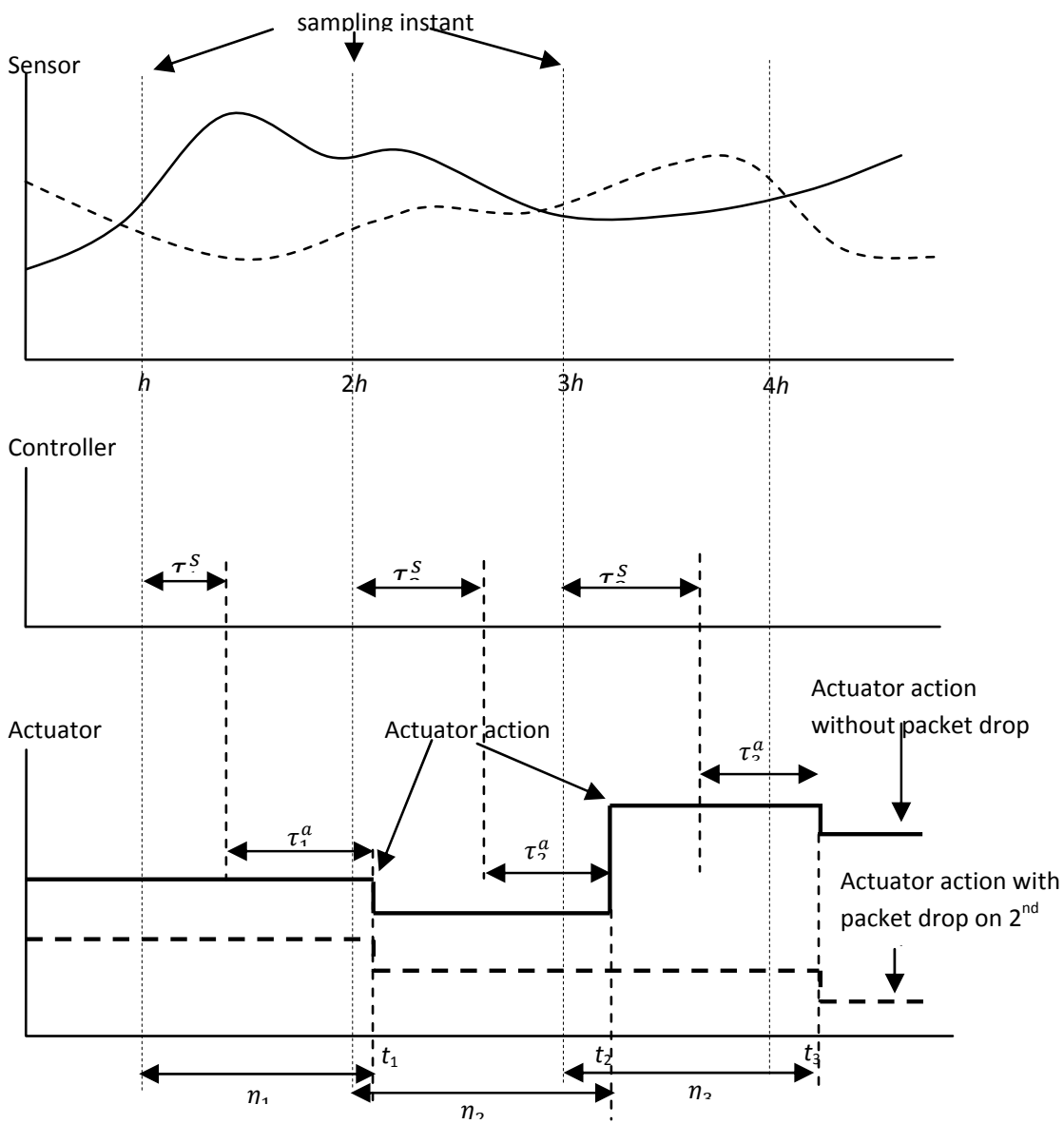

Figure 2. Timing diagram

In a similar way as in [5], let us represent $t_{k}-\eta_{k}$ in (9) as

$t_{k}-\eta_{k}=t-\eta_{m}-\eta(t)$,

where $\eta(t)=t-t_{k}+\eta_{k}-\eta_{m}, t_{k} \leq t<t_{k+1}$. It is obviously that

$0 \leq \eta(t)<t_{k+1}-t_{k}+\left(\eta_{k}-\eta_{m}\right)$

By substituting (11) into (13), we have

$$
\begin{aligned}
& 0 \leq \eta(t)<\left(\delta_{k+1}+1\right) h+\eta_{k+1}-\eta_{k}+\left(\eta_{k}-\eta_{m}\right) \\
& 0 \leq \eta(t) \leq \kappa
\end{aligned}
$$

where $\kappa=(\bar{\delta}+1) h+\eta_{M}-\eta_{m}, \bar{\delta}$ denotes the maximum number of packet dropouts. We illustrate the timing diagram of NCS described above in Figure 2.

By substituting (12) into (9) we have

$$
\begin{aligned}
& \dot{x}(t)=A x(t)+B K x\left(t-\eta_{m}-\eta(t)\right)+E w(t), \\
& y(t)=C x(t)+D K x\left(t-\eta_{m}-\eta(t)\right)+F w(t),
\end{aligned}
$$




\section{Controller Design}

In this section, we solve the problem of $H_{\infty}$ state feedback controller design for NCS described in previous section. The problem we address here stated as follows: Design a state feedback controller in the form of (8) such that the closed-loop system (15) is asymptotically stable with $H_{\infty}$ disturbance attenuation level $\gamma$.

The main result of the present paper stated in the following theorem.

Theorem 1. Consider the NCS in Figure1. For given scalars $\rho_{i},(i=2,3,4), \eta_{m}, \eta_{M}, h$, and $\bar{\delta}$. There exists a state feedback controller in the form of (8) such that the closed-loop system in (15) either,

(i). asymptotically stable with $w=0$, or

(ii). asymptotically stable with $\mathrm{H}_{\infty}$ disturbance attenuation level $\gamma(w \neq 0)$

if there exist matrices $\bar{P}=\bar{P}^{T}>0, \bar{Q}_{2}=\bar{Q}_{2}^{T}>0, \bar{R}_{i}=\bar{R}_{i}^{T}>0(i=1,2,3)$, non-singular matrix $X$, and $\bar{L}_{i}, \bar{M}_{i}, \bar{N}_{i}(i=1, \ldots, 4)$ are free matrices satisfying,

$$
\left[\begin{array}{ccc}
\Omega_{1}+\Omega_{2}+\Psi_{4} & \Omega_{5} & \Omega_{3} \\
* & \Omega_{4} & 0 \\
* & * & -I
\end{array}\right]<0
$$

where

$$
\begin{aligned}
& \Omega_{1}=\left[\begin{array}{ccccc}
\bar{Q}_{2}+A X^{T}+X A^{T} & \rho_{2} X A^{T} & B \bar{K}+\rho_{3} X A^{T} & \Omega_{114} & E \\
* & -\bar{Q}_{2} & \rho_{2} B \bar{K} & -\rho_{2} X^{T} & \rho_{2} E \\
* & * & \rho_{3} B \bar{K}+\rho_{3} \bar{K}^{T}{ }_{B} T & \Omega_{134} & \rho_{3} E \\
* & * & * & \Omega_{144} & \rho_{4} E \\
* & * & * & * & 0
\end{array}\right], \\
& \Omega_{114}=\bar{P}-X^{T}+\rho_{3} X A^{T}, \Omega_{134}=-\rho_{3} X^{T}+\rho_{4} \bar{K}^{T} B^{T} \\
& \Omega_{144}=v \bar{R}_{1}+\eta_{m} \bar{R}_{2}+\kappa \bar{R}_{3}-\rho_{4} X^{T}-\rho_{4} X \text {, } \\
& \Omega_{2}=\left[\begin{array}{ccccc}
\bar{L}_{1}+\bar{L}_{1}^{T}+\bar{M}_{1}+\bar{M}_{1}^{T} & \bar{L}_{2}^{T}-\bar{M}_{1}+\bar{M}_{2}^{T}+\bar{N}_{1} & -\bar{L}_{1}+\bar{L}_{3}^{T}+\bar{M}_{3}^{T}-\bar{N}_{1} & \bar{L}_{4}^{T}+\bar{M}_{4}^{T} & 0 \\
* & -\bar{M}_{2}-\bar{M}_{2}^{T}+\bar{N}_{2}+\bar{N}_{2}^{T} & -\bar{L}_{2}-\bar{M}_{3}^{T}-\bar{N}_{2}+\bar{N}_{3}^{T} & -M_{4}^{T}+\bar{N}_{4}^{T} & 0 \\
* & * & -\bar{L}_{3}-\bar{L}_{3}^{T}-\bar{N}_{3}-\bar{N}_{3}^{T} & -\bar{L}_{4}^{T}-\bar{N}_{4}^{T} & 0 \\
* & * & * & 0 & 0 \\
* & * & * & * & 0
\end{array}\right], \\
& \Omega_{5}=\left[\begin{array}{ccc}
\bar{L}_{1} & \bar{M}_{1} & \bar{N}_{1} \\
\bar{L}_{2} & \bar{M}_{2} & \bar{N}_{2} \\
\bar{L}_{3} & \bar{M}_{3} & \bar{N}_{3} \\
\bar{L}_{4} & \bar{M}_{4} & \bar{N}_{4} \\
0 & 0 & 0
\end{array}\right], \\
& \Psi_{4}=\operatorname{diag}\left\{0,0,0,0,-\gamma^{2} I\right\}, \Omega_{4}=\operatorname{diag}\left\{-v^{-1} \bar{R}_{1},-\eta_{m}^{-1} \bar{R}_{2},-\kappa^{-1} \bar{R}_{3}\right\}, \\
& \Omega_{3}=\left[\begin{array}{lllll}
C X^{T} & 0 & D \bar{K} & 0 & F
\end{array}\right]^{T}, v=\eta_{m}+\kappa \text { and } \kappa=(\bar{\delta}+1) h+\eta_{M}-\eta_{m} .
\end{aligned}
$$

If condition in (16) feasible, a desired controller gain matrix is given by

$$
K=\bar{K}\left(X^{T}\right)^{-1}
$$


Proof. By comparing system (15) and system (1) satisfying (2), according to Lemma 2, we know that system (15) is asymptotically stable with $H_{\infty}$ disturbance attenuation level $\gamma$ if there exist matrices $P=P^{T}>0, Q_{2}=Q_{2}{ }^{T}>0, R_{i}=R_{i}{ }^{T}>0(i=1,2,3)$, and $G_{i}, L_{i}, M_{i}, N_{i},(i=$ $1, \ldots, 4)$ are free matrices satisfying,

$$
\left[\begin{array}{cc}
\phi_{4}+\phi_{2}+\phi_{2}^{T}+\Psi_{3}+\Psi_{4}+\phi_{3} \phi_{3}^{T} & \Psi_{5} \\
* & \phi_{1}
\end{array}\right]<0
$$

where

$$
\begin{aligned}
& \phi_{4}=\left[\begin{array}{ccccr}
Q_{2} & 0 & 0 & P & 0 \\
* & -Q_{2} & 0 & 0 & 0 \\
* & * & 0 & 0 & 0 \\
* & * & * & v R_{1}+\eta_{m} R_{2}+\kappa R_{3} & 0 \\
* & * & * & * & 0
\end{array}\right] \\
& v=\eta_{m}+\kappa, \kappa=(\bar{\delta}+1) h+\eta_{M}-\eta_{m}, \\
& \phi_{2}=\phi_{21} \Psi_{22}, \phi_{21}=\left[\begin{array}{llllll}
A & 0 & B K & -I & E
\end{array}\right]^{T}, \quad \phi_{3}=\left[\begin{array}{lllll}
C & 0 & D K & 0 & F
\end{array}\right]^{T}, \\
& \phi_{1}=\operatorname{diag}\left\{-v^{-1} R_{1},-\eta_{m}^{-1} R_{2},-\kappa^{-1} R_{3}\right\}, \text { and } \Psi_{22}, \Psi_{3}, \Psi_{4}, \Psi_{5} \text { are given in (3). }
\end{aligned}
$$

By Lemma 1 (Schur complement), (18) is equivalent to

$$
\left[\begin{array}{ccc}
\phi_{4}+\phi_{2}+\phi_{2}^{T}+\Psi_{3}+\Psi_{4} & \Psi_{5} & \phi_{3} \\
* & \phi_{1} & 0 \\
* & * & -I
\end{array}\right]<0
$$

Define $G_{l}=G_{0}$ and $G_{i}=\rho_{i} G_{0}\left(i=2,3,4 ; \rho_{i} \neq 0\right)$, (19) become

$$
\left[\begin{array}{ccc}
\phi_{1}+\bar{\phi}_{2}+\bar{\phi}_{2}^{T}+\Psi_{3}+\Psi_{4} & \Psi_{5} & \phi_{3} \\
* & \phi_{1} & 0 \\
* & * & -I
\end{array}\right]<0
$$

where

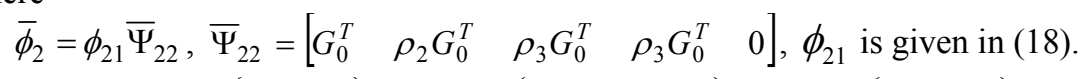

Define $J=\operatorname{diag}\left\{J_{1}, J_{2}, I\right\}, J_{1}=\operatorname{diag}\{X, X, X, X, I\}, J_{2}=\operatorname{diag}\{X, X, X\}, X=G_{0}^{-1}$.

Applying congruence transformation [13] to (20) by $J$, we get

$$
\left[\begin{array}{ccc}
J_{1}\left(\phi_{4}+\bar{\phi}_{2}+\bar{\phi}_{2}^{T}+\Psi_{3}+\Psi_{4}\right) J_{1}^{T} & J_{1} \Psi_{5} J_{2}^{T} & J_{1} \phi_{3} \\
* & J_{2} \phi_{1} J_{2}^{T} & 0 \\
* & * & -I
\end{array}\right]<0
$$

Simplifying (21) together with the change of variables defined by,

$$
\begin{aligned}
& \bar{L}_{i}=X L_{i} X^{T}, \bar{M}_{i}=X M_{i} X^{T}, \bar{N}_{i}=X N_{i} X^{T}(i=1, \ldots, 4), \\
& \bar{R}_{i}=X R_{i} X^{T}(i=1,2,3), \bar{Q}_{2}=X Q_{2} X^{T}, \bar{K}=K X^{T}, \bar{P}=X P X^{T}
\end{aligned}
$$


then (16) is obtained, and thus this proof is completed.

Worthy note that, Theorem 1 in this paper reduces the number of variables appeared in Theorem 1 in [16], those are $L_{5}, M_{5}$, and $N_{5}$.

\section{Numerical Example}

In this section, we provide an example to show the applicability of the proposed controller design. The system matrices $A, B, C, D, E, F$ in (4) are given as follows,

$$
A=\left[\begin{array}{ll}
0 & 1 \\
1 & 2
\end{array}\right], B=\left[\begin{array}{l}
0 \\
1
\end{array}\right], E=\left[\begin{array}{l}
0.3 \\
0.5
\end{array}\right], C=\left[\begin{array}{ll}
1 & 0
\end{array}\right], \mathrm{D}=0.3 \text { and } \mathrm{F}=0.5
$$

The eigenvalues of $A$ are -0.4142 and 2.4142 , thus the above systems is unstable.

Our objective is to design a state feedback controller in the form of (8) such that the closedloop system is asymptotically stable with an $H_{\infty}$ disturbance attenuation level $\gamma$. It is assumed that, minimum delay , $\eta_{m}=10 \mathrm{~ms}$, maximum delay, $\eta_{M}=40 \mathrm{~ms}$, sampling period, $h=10 \mathrm{~ms}$, and $\rho_{2}=\rho_{3}=\rho_{4}=1$.

First, we assume there is no packet drop, that is $\bar{\delta}=0$. This means that all data packet succesfully transmitted to the $\mathrm{ZOH}$.

By solving LMI in Theorem 1, that is minimizing $\gamma$ in (16), we get the following results

$$
X=\left[\begin{array}{cc}
1.0162 & -1.0284 \\
0.5483 & 3.8142
\end{array}\right], \bar{K}=\left[\begin{array}{ll}
-4.4193 & -19.1791
\end{array}\right]
$$

So, according to (17), the state feddback gain controller in (8) is given by

$$
K=\bar{K}\left(X^{T}\right)^{-1}=\left[\begin{array}{ll}
-8.2389 & -3.8439
\end{array}\right]
$$

and the minimum guaranteed $H_{\infty}$ performance is $\gamma=0.638$.

Now, we assume the maximum packet dropout, $\bar{\delta}=2$.

We obtain the controller gain,

$$
K=\left[\begin{array}{ll}
-7.0482 & -3.7757
\end{array}\right]
$$

and the minimum guaranteed $H_{\infty}$ performance is $\gamma=0.657$. The results above show that the $H_{\infty}$ performance of the NCS without packet drop is better than the NCS with packet drop.

For the next experiment, we consider the effect of $\rho_{2}, \rho_{3}$, and $\rho_{4}$ to the obtained controller gain $K$. By solving LMI in Theorem 1 with $\eta_{m}=10 \mathrm{~ms}, \eta_{M}=40 \mathrm{~ms}, h=10 \mathrm{~ms}$, and $\bar{\delta}=2$, the obtained controller gain $K$ and the minimum guaranteed $H_{\infty}$ performance $\gamma$ are listed in Table 1 for different values of $\rho_{2}, \rho_{3}$, and $\rho_{4}$.

For the simulation of the closed-loop system, the initial state is assumed to be $\left[\begin{array}{ll}1 & 0.5\end{array}\right]^{T}$. Figure 3 show the state response for different values of $K$ (the closed-loop system is asymptotically stable with the above controller gain). During the simulation, network induced delays is generated randomly within their range according to the above assumption. 

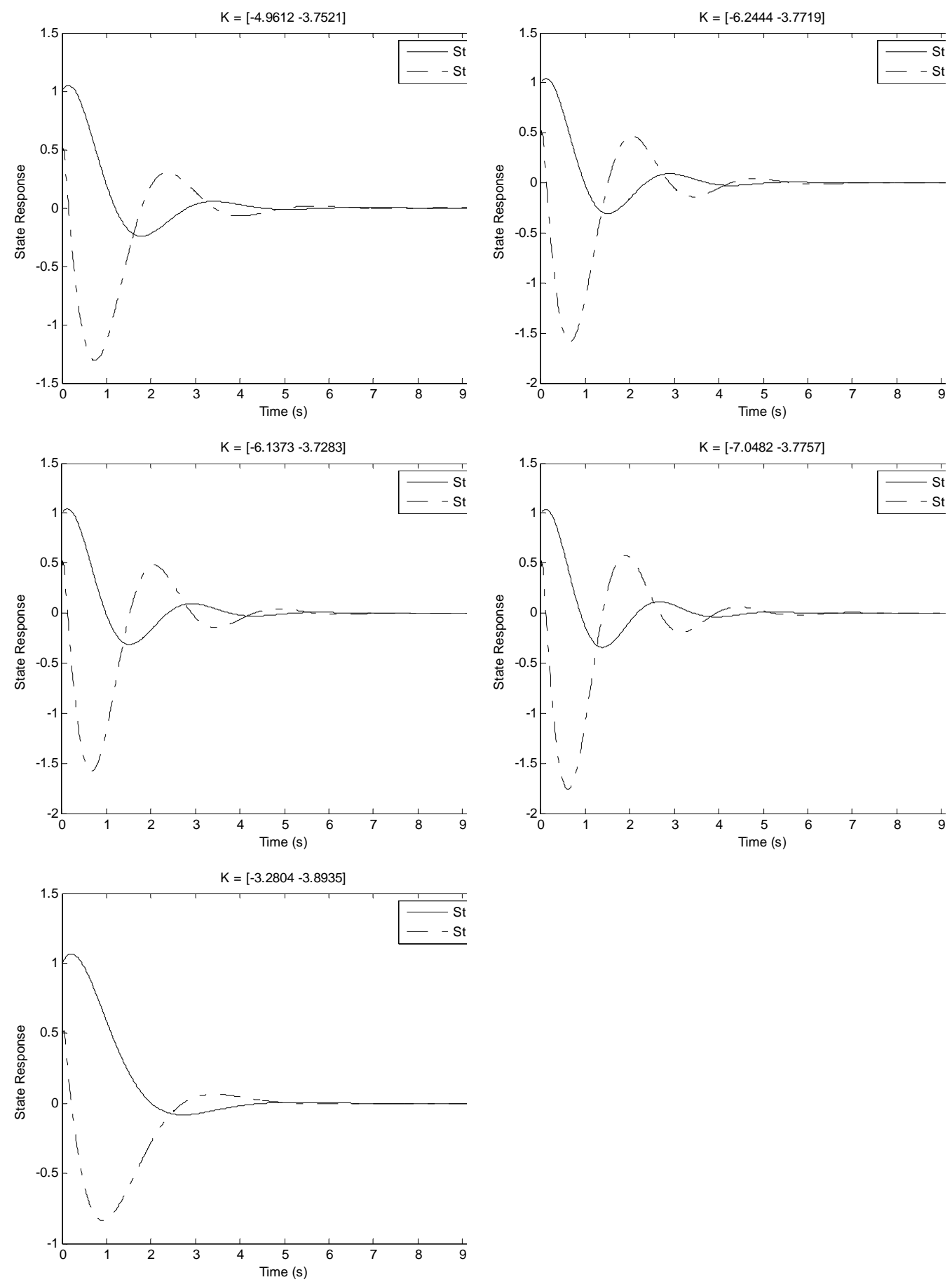

Figure 3. State response without input disturbance for different values of $K$

Next, we select input disturbance signal as follows:

$$
w(t)=\left\{\begin{array}{c}
\sin t, \quad 5 \leq t \leq 15 s, \\
0, \text { otherwise }
\end{array}\right.
$$


Wrastawa Ridwan, et al.

Table 1 . The obtained $K$ and $\gamma$ for different values of $\rho_{2}, \rho_{3}$, and $\rho_{4}$

\begin{tabular}{|c|c|c|c|c|c|c|}
\hline No. & $\rho_{2}$ & $\rho_{3}$ & $\rho_{4}$ & $\gamma$ & K & \\
\hline 1. & $10^{-3}$ & $10^{-3}$ & 1 & 0.743 & {$[-4.9612$} & $-3.7521]$ \\
\hline 2. & $10^{-3}$ & $10^{-3}$ & $10^{-3}$ & infeasible & - & \\
\hline 3. & $10^{-3}$ & 1 & $10^{-3}$ & infeasible & - & \\
\hline 4. & $10^{-3}$ & 1 & 1 & 0.68 & {$[-6.2444$} & -3.7719] \\
\hline 5. & 1 & $10^{-3}$ & $10^{-3}$ & infeasible & - & \\
\hline 6. & 1 & $10^{-3}$ & 1 & 0.681 & {$[-6.1373$} & $-3.7283]$ \\
\hline 7. & 1 & 1 & $10^{-3}$ & infeasible & - & \\
\hline 8. & 1 & 1 & 1 & 0.657 & {$[-7.0482$} & $-3.7757]$ \\
\hline 9. & 5 & 10 & 100 & 1.154 & {$[-3.2804$} & -3.8935] \\
\hline 10. & $10^{-5}$ & $10^{-5}$ & $10^{-5}$ & infeasible & - & \\
\hline
\end{tabular}

Figure 4 depict the state response under above disturbance for $K=\left[\begin{array}{ll}-7.0482 & -3.7757\end{array}\right]$, while Figure 5 depict the state response for $\mathrm{K}=\left[\begin{array}{ll}-3.2804 & -3.8935\end{array}\right]$.

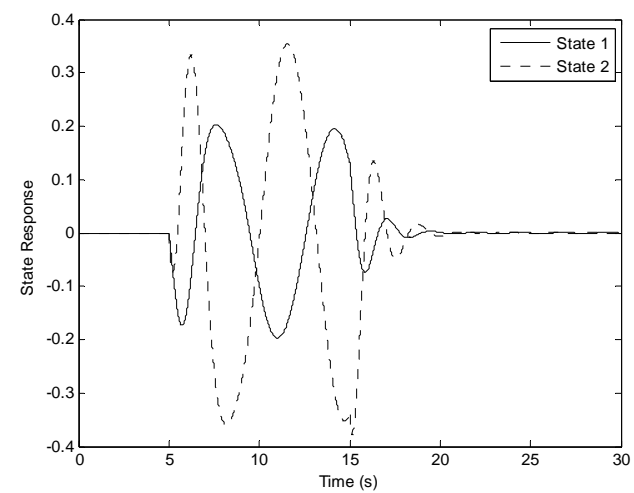

Figure 4. State response with input disturbance for $K=\left[\begin{array}{ll}-7.0482 & -3.7757\end{array}\right]$

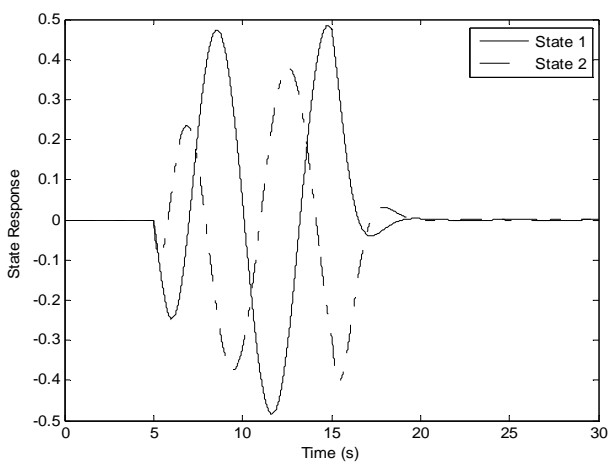

Figure 5. State response with input disturbance for $K=\left[\begin{array}{ll}-3.2804 & -3.8935\end{array}\right]$

From Table 1, one can see that the the smallest $\gamma$ when $\rho_{2}=\rho_{3}=\rho_{4}=1$, where $K=$ $\left[\begin{array}{ll}-7.0482-3.7757\end{array}\right]$. These result shown when comparing Figure 4 (smallest $\gamma$ ) and Figure 5 (biggest $\gamma$ ), where the smallest $\gamma$ yields the smallest the amplitud of the state response. 


\section{Conclusions}

This paper presented the design of state feedback $H_{\infty}$ controller of NCS which guaranteed the closed-loop system to be asymptotically stable. Two network features are considered when modelling the NCS: signal transmission delay and data packet dropout. The desired state feedback controller could be obtained by solving an LMI condition. The effectiveness of the proposed method has been verified by numerical example.

\section{References}

[1] Rachana A. Gupta, and Mo-Yuen Chow, "Networked control system: overview and research trends," IEEE Trans. Industrial Electronics, vol. 57, no. 7, July 2010.

[2] F-Y. Wang, and D. Liu, Eds., Networked Control Systems: Theory and Application, London, Springer-Verlag, 2008.

[3] J. P. Hespanha, P. Naghshtabrizi, and Y. Xu, "A survey of recent results in networked control systems," Proc. IEEE, vol. 95, no. 1, pp. 138-162, Jan. 2007.

[4] J. Dai, "A delay system approach to networked control sytems with limited communication capacity,"Journal of The Franklin Institute, vol. 347, pp. 1334-1352, 2010.

[5] H. Gao, T. Chen, and J. Lam, "A new delay system approach to networked-based control," Automatica, vol.44, pp.39-52, 2008.

[6] X-M. Zhang, and Q-L. Han, "A delay decomposition approach to $\mathrm{H}_{\infty}$ control of networked control systems," European Journal of Control, vol. 5, pp. 523-533, 2009.

[7] Q.C. Zhong, Robust Control of Time-Delay Systems, London, Springer-Verlag, 2006.

[8] S.I. Niculescu, Delay Effects on Stability: A Robust Control Approach, Heidelberg, Springer, 2001.

[9] Y. Xia, M. Fu, and P. Shi, Analysis and synthesis of dynamical systems with time-delays, Berlin, Germany: Springer-Verlag, 2009.

[10] M. Wu, Y. He, and J. H. She, Stability Analysis and Robust Control of Time-Delay Systems, London, Science Press Beijing and Springer-Verlag, 2010.

[11] J. Lam, H. Gao, and C. Wang, "Stability analysis for continous system with two additive time-varying delays components," System Control Letters, vol.56, pp.16-24, 2007.

[12] R. Dey, G. Ray, S. Ghosh, and A. Rakhsit, "Stability analysis for continuous system with additive time-varying delays: a less conservative result," Applied Mathematics and Computation, vol. 215, pp. 3740-3745, 2010.

[13] Matthew C. Turner, and Declan G. Bates, Eds., Mathematical Methods for Robust and Nonlinear Control, ser. Lecture Notes in Control and Information Sciences. Berlin, Germany: Springer, 2007, vol. 367.

[14] S. L. Boyd, L. E. Ghaoui, E. Feron, and V. Balakrishnan, Linear Matrix Inequalities in Systems and Control Theory, Philadelphia, SIAM, 1994.

[15] W. Ridwan and R. T. Bambang, "An LMI approach to $\mathrm{H}_{\infty}$ performance analysis of continuous-time systems with two additive time-varying delays," ITB Journal of Engineering Science, vol.43 (2), pp. 127-138, 2011.

[16] W. Ridwan and R. T. Bambang, " $\mathrm{H}_{\infty}$ Controller Synthesis for Networked Control Systems with Time Delay System Approach", Int. Conf. on Electrical Engineering and Informatics, Bandung, July 2011, pp. 714-718. 


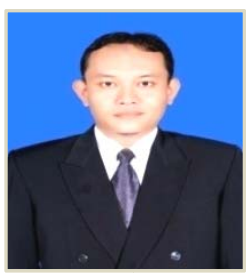

Wrastawa Ridwan was born in Manado, Indonesia, on February 5, 1979. $\mathrm{He}$ received his engineering degree in Control Systems from Sepuluh Nopember Institute of Technology, Surabaya, Indonesia in 2002. Since 2005, he has been working as a lecturer in the Department of Electrical Engineering Gorontalo State University, Indonesia. He received his Master degree from Bandung Institute of Technology, majoring in Control and Intelligent Systems in 2011. His research interests are control systems, networked control system, and intelligent systems.

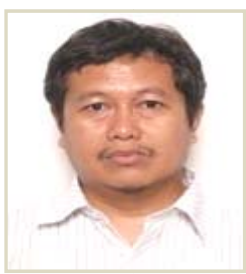

Bambang Riyanto Trilaksono was born in Banyuwangi, Indonesia, on November 15, 1962. He received his engineering degree in Electrical Engineering from Bandung Institute of Technology, Indonesia in 1986. He received the master and doctorate degree from Waseda University, Japan in 1991 and 1994 respectively. Now, he is a Professor at Department of Electrical Engineering, Bandung Institute of Technology, Indonesia. He is a well known researcher in control engineering in Indonesia as well as in international control engineering society. His research interests include robust control, intelligent control \& intelligent systems, discrete event systems, control applications, telerobotics, embedded control systems and robotics. 